\title{
The representation of explicit motor sequence knowledge
}

\author{
Robert Knee and Sean Thomason \\ University of Virginia, Charlottesville, Virginia \\ JAMES ASHE \\ University of Minnesota, Minneapolis, Minnesota \\ AND \\ Daniel T. WillinghaM \\ University of Virginia, Charlottesville, Virginia
}

\begin{abstract}
Much research has investigated the representation of implicitly learned motor sequences: Do subjects learn sequences of stimuli, responses, response locations, or some combination? Most of the work on this subject indicates that when sequences are learned implicitly, it is in terms of response locations. The present work investigated the representation of explicitly learned motor sequences. In four experiments, we found consistent evidence that explicitly learned sequences are represented in terms of stimulus locations. This conclusion held true for both self-report measures (subjects said that they learned stimuli) and performance measures, but when stimuli changed, performance degraded. We interpret these data in a multiple-memory-systems framework.
\end{abstract}

The learning of sequences is a pervasive and crucial aspect of motor skill acquisition. The question of how such sequences are learned has therefore been one of enduring concern.

A fundamental distinction has been drawn between the learning of sequences in implicit and explicit tasks. A task is implicit if knowledge is tested indirectly via performance, and the subject remains unaware that he or she has learned. A task is explicit if knowledge is queried directly - usually via recognition or recall-and the subject is aware that learning has taken place (Graf \& Schacter, 1985).

Many researchers studying sequence learning have favored the serial response time task (SRTT; Nissen \& Bullemer, 1987) because implicit and explicit versions of the task are so similar. In the SRTT, the subject performs a speeded, continuous four-choice response time (RT) task. For some of the trials, the stimuli appear in a repeating sequence (frequently a 12 -unit sequence is used). In the implicit version of the task, the experimenter does not inform the subject about the sequence, and sequence knowledge is assessed by an RT difference to the sequenced and the random stimuli. In the explicit version of the task, the subject is informed about the sequence, and explicit knowledge is measured by recognition or recall.

Many researchers have speculated that implicit and explicit versions of the task are supported by separate memory systems (Reber \& Squire, 1998; Willingham, Nissen, \& Bullemer, 1989); here, we use the terms procedural and declarative (N. J. Cohen \& Squire, 1980) to refer to the memory systems that are hypothesized to support implicit and explicit versions of the task, respectively.

Support for the separability of procedural and declarative memory has been twofold. First, the two memory systems appear to be supported by different networks of neural structures, with procedural memory relying on the basal ganglia, parietal and motor cortices, and perhaps the prefrontal cortex. In contrast, declarative memory relies on the medial temporal lobe, diencephalon, and areas of prefrontal and parietal lobes that may or may not overlap with those supporting procedural memory (see, e.g., Gabrieli, 1998; Squire, 1992; Willingham, 1998). The second source of evidence supporting the separability of procedural and declarative memory is the dissociation of awareness. Subjects performing the implicit version of the task frequently remain unaware of the sequence, although their performance indicates learning has occurred. Subjects performing the explicit version are of course aware of the sequence (e.g., Reed \& Johnson, 1994; Willingham et al., 1989).

A third potential source of evidence for the separability of procedural and declarative learning would be that procedural and declarative memory systems learn different things. Distinctions of memory systems in the rat have included not only anatomic dissociations, but also evidence that each memory system is biased toward learning different sources of information in the environment. In the case

D. T.Willingham, willingham@virginia.edu 
of the SRTT, we might ask what a subject learns - in other words, what is the learned sequence a sequence of? A sequence of stimuli? Of responses? This question has been addressed by a number of researchers in the case of procedural memory, and the bulk of the evidence seems to favor a representation of response locations (Jiménez \& Méndez, 1999; Nattkemper \& Prinz, 1997; Willingham, 1999; Ziessler, 1994, 1998; Ziessler \& Nattkemper, 2001). That is, the subject learns where to respond independent of the particular stimuli evincing the responses or the motor effector executing the responses.

Willingham (1999) provided evidence supporting this conclusion. Subjects performed the implicit SRTT using an incompatible stimulus-response mapping. After subjects learned the sequence, they transferred to a version of the task using a compatible mapping. Some subjects saw the same sequence of stimuli (which now called for new responses because the mapping had changed). These subjects showed no transfer at all. Other subjects saw a new sequence of stimuli that led to the same response locations used during training; these subjects showed excellent transfer. Other work has demonstrated that learning in the implicit version of the SRTT transfers across effectors when the stimulus locations remain the same (A. Cohen, Ivry, \& Keele, 1990; Grafton, Hazeltine, \& Ivry, 1998; Keele, Jennings, Jones, Caulton, \& Cohen, 1995).

The results of some experiments have indicated that implicit learning may be perceptually based, but in some of these results, declarative knowledge may have made a contribution to performance (Howard, Mutter, \& Howard, 1992; Koch \& Hoffmann, 2000; Ziessler \& Nattkemper, 2001; but see Remillard, 2003). In other experiments (Goschke, 1998; Mayr, 1996) the putatively perceptual knowledge may have been due to learning sequences of eye movements (see Grafton, Salidis, \& Willingham, 2001).

A great deal of attention has focused on the nature of procedural learning in the SRTT, but virtually none has addressed the nature of declarative learning. When subjects explicitly learn a sequence, what do they learn? One of us (Willingham, 1998) has suggested that in explicit versions of the task, subjects learn a sequence of stimulus positions in contrast with the response locations learned in the implicit version. The experiments reported here were designed to collect data relevant to this proposal.

\section{EXPERIMENT 1}

In this experiment, subjects explicitly learned the sequence in the SRTT and then were asked an open-ended question regarding whether they had learned a sequence of stimuli, response keys, or finger movements.

\section{Method}

Subjects. Twenty-five undergraduate students (5 male) from the University of Virginia participated in return for research credit in an introductory psychology course. The subjects all had normal hearing and normal or corrected-to-normal vision, as determined by selfreport. Average age was 18.2 years (range, 18-19).

Stimuli and Apparatus. Stimuli were presented on a Dell 17-in. color monitor, controlled by a Dell Pentium PC. Subjects sat approximately $50 \mathrm{~cm}$ from the screen. Stimuli were generated and responses were collected using the E-Prime Version 1.0 software package (Psychology Software Tools, Pittsburgh, PA). Four stimulus locations were represented by on-screen boxes - each measuring approximately $2.5 \mathrm{~cm}$ square - arrayed horizontally and centered vertically. The distance from the leftmost to the rightmost stimulus was $24.1 \mathrm{~cm}\left(26.7^{\circ}\right.$ visual angle $)$. The boxes were approximately equidistant from each other and from the screen's edge. Subjects responded to a stimulus appearing in one of the boxes. The stimulus was a solid circle, approximately $1.8 \mathrm{~cm}$ in diameter (3.6 visual angle).

Responses were collected on four keys on the Serial Response Box (Model 200a, Psychology Software Tools). These four keys are arrayed horizontally, with a center-to-center distance of approximately $0.9 \mathrm{~cm}$. The fifth, rightmost key on the box was not used.

Procedure. Subjects were told that on each trial a circle would appear in one of the boxes and that they should respond by pressing the corresponding key. Neither speed nor accuracy was stressed. The leftmost box mapped to the leftmost key, the next box to the right mapped to the next key to the right and so on, but the experimenter did not label the locations or keys when giving these instructions; rather, the experimenter pointed to the keys and boxes. The experimenter demonstrated the correct finger placement by placing the middle finger and index finger of each hand on the keys.

Subjects were also told that the stimuli would appear in a repeating sequence 12 units long and that they should focus on learning the sequence.

On each trial a stimulus appeared and remained visible until the subject made a correct response, whereupon the stimulus was extinguished and the next stimulus appeared with the next screen refresh (refresh rate was $72 \mathrm{~Hz}$ ). Errors and anticipations were signaled by a 300-msec tone. In order to assist declarative learning, after each sequence presentation the boxes were extinguished and a fixation cross immediately appeared at the center of the screen for $750 \mathrm{msec}$. Each trial block was composed of 96 stimuli, or eight repetitions of the sequence. The starting point of the sequence was the same across blocks. Subjects performed four trial blocks for a total of 32 repetitions of the sequence.

Each subject saw a different 12-unit sequence taken from a list of 583 possible sequences that followed these rules: Each stimulus location appeared three times; the same stimulus could not appear on successive trials, and the sequence could not contain runs (e.g., 4321) or trills (e.g., 1414) of four stimuli.

After training, the experimenter told the subjects, "Please describe in your own words how you went about learning the pattern. Use any technique, analogies, or references you would like.” The experimenter recorded verbatim what the subject reported. The experimenter then asked the subject to recall the sequence and recorded the response. When the subject had completed the recall, the experimenter reminded the subject of what he or she had said about the technique that was used in learning the sequence and offered the subject the chance to change his or her description. We offered the chance to change the description because we thought that actually recalling the sequence might give the subject more insight into the technique he or she had used to explicitly learn it.

\section{Results and Discussion}

Each subject was first asked about his or her strategy in learning the sequences. Subjects were coded into one of three categories: The screen group made some explicit mention of memorizing screen positions or stimuli; the response group made some mention of memorizing key locations or finger movements, and the switch group described a strategy that involved some combination of screen and responses or reported that they switched between these strategies during the task.

When queried about technique, 12 subjects spontaneously referred to coding either screen positions or key positions. Thirteen reported generating their own codes 
(e.g., digits). These 13 subjects were asked to what this code corresponded, and the subject was categorized as belonging to the screen, response, or switch group, as appropriate.

Twenty-two subjects were categorized as memorizing stimuli, 2 subjects were categorized as memorizing responses, and 1 subject switched. Statistical analysis is hardly necessary, but a chi-square analysis showed a reliable difference among the categories $\left[\chi^{2}(2, N=25)=\right.$ $33.68, p<.001]$.

Of the 13 subjects using a labeling strategy, 6 used digits, 6 used tones, and 1 subject reported using a conjunction of numbers and tones. Both of the subjects who memorized response locations used the digits labels, but we do not regard this as especially noteworthy because of the small number of observations.

Subjects were next asked to produce the sequence via free recall, which was scored by summing all correct sequence segments. A recalled segment had to be at least three units long, however, to contribute since the probability of any two positions (e.g., 32) matching the sequence by chance was quite high. Correct segments did not need to be consecutive to contribute to the total score. For example, suppose the subject saw the sequence 413214232341 and recalled 3214321234 . The score would be 7 , because 3214 occurs in the sequence and so does 234; the subject's repetition of 321 would not count a second time.

Mean recall was 10.8 positions $(S D=2.5)$ correct out of 12 possible. Eighteen subjects got 12 positions correct; 3 got 6 or fewer. Thus, most subjects were able to learn the sequence effectively.

After recalling the sequence, subjects were again queried about their strategy. Although some amplified their earlier response, none substantially changed their description of their strategy.

This experiment provides clear-cut evidence regarding what subjects believe they learn and attend to when they learn an explicit motor sequence. In Experiment 2, we sought a measure that did not rely on self-report.

\section{EXPERIMENT 2}

In Experiment 2, subjects were again asked to explicitly learn a sequence, but this time the stimulus-response mapping was incompatible. After learning the sequence, the subjects took a recognition test of the sequence, but the mapping was compatible. Thus, the sequence during the recognition task could either entail the same stimuli that was seen during training (but different responses), the same responses that were executed during training (but different stimuli), or altogether novel stimuli. The question of interest was whether subjects would recognize targets that used the same stimuli, the same responses, or both. Note that this design is conceptually similar to that used by Willingham (1999), the results of which indicated that implicit sequence learning is based in response locations.

\footnotetext{
Method

Subjects. Twenty-four undergraduate students (11 male) from the University of Virginia participated in return for research credit
}

in an introductory psychology course. All subjects reported normal hearing and normal or corrected-to-normal vision. Average subject age was 18.9 years (range, $18-21$ years).

Stimuli and Apparatus. The stimuli and apparatus were the same as those in Experiment 1, except that the mapping between stimuli and responses was incompatible. Instead of responding using the button directly below the stimulus, subjects were asked to respond by using the key one position to the right. Thus, by designating stimulus positions and keys from left to right as $1-4$, when Stimulus 1 appeared, they were to press Key 2, and so on. The exception was Stimulus Position 4, for which subjects were to "wrap around" and press Key 1.

Procedure. The procedure for this experiment was identical to that of Experiment 1 except that the interview was replaced by a recognition task.

Subjects were told that during the recognition task they would respond to a six-unit stimulus segment as they did in the SRT task, but that they should use a compatible mapping for this phase of the experiment. After responding to the six stimuli, they should make a judgment of whether the segment was taken from the sequence used during training by employing a rating scale of 1 to 4 . Each rating was labeled as follows: (1) very sure the sequence is new, (2) fairly sure the sequence is new, (3) fairly sure the sequence is the old one, (4) very sure the sequence is the old one. The subjects recorded their ratings using a $\mathrm{PC}$ keyboard.

Each subject saw a total of 24 six-unit sequences. Six were segments of the training stimulus sequence, six were segments of the response sequence, and 12 of the sequences were novel. The novel sequences were selected randomly from the corpus of 12-unit sequences with the constraint that they not overlap perfectly with any segment of the stimulus or response sequence. Partial overlap was permissible.

\section{Results and Discussion}

The mean recognition ratings were as follows: novel $(M=2.17, S E M=0.09)$, stimulus $(M=2.77, S E M=$ $0.12)$, and response $(M=2.77, S E M=0.12)$. A repeated measures ANOVA showed that recognition scores were reliably different $\left[F(2,22)=7.73, M S_{\mathrm{e}}=0.16, p<.01\right]$. Follow-up paired-samples $t$ tests showed that the ratings for the response sequences did not differ from the ratings for the novel sequences $[t(23)=0.52, S E=0.89, p>$ $.20]$, but that ratings for the stimulus sequence differed reliably from those of the other two groups [both $t \mathrm{~s}(23)>$ 3.7 , both $p \mathrm{~s}<.001]$.

These data are consistent with the hypothesis that explicit learning is related to stimuli, whereas previous literature indicates that implicit learning is related to response locations. There are, however, some differences between the present implementation of the SRTT and that of prior work. In Experiment 3, we sought to replicate Experiment 1 while making the paradigm more comparable to prior investigations of implicit learning.

\section{EXPERIMENT 3}

\section{Method}

Subjects. Fifty-seven undergraduate students from the University of Virginia participated in return for research credit in an introductory psychology course. All subjects reported normal hearing and normal or corrected-to-normal vision. Average subject age was 18.8 years (range, 18-23 years). Gender was not recorded for this experiment.

Procedure. The stimuli, responses, and procedure were identical to those of Experiment 1, with three small changes designed to make 
this experiment more comparable to previous implicit SRTT experiments. Experiment 1 used a very brief response-to-stimulus interval (RSI); this experiment used a 500-msec RSI. In Experiment 1, a crosshair appeared at the end of each sequence to improve explicit learning; the crosshair was omitted in Experiment 3 and the RSI was set to $500 \mathrm{msec}$, so that nothing marked the beginning or end of the sequence. In Experiments 1 and 2, reference was made in the instructions to a sequence of stimuli, which could have influenced subjects to learn the stimulus sequence rather than the response sequence. In this experiment, subjects were informed that there was "a sequence embedded in the task" that they were to learn; it was not referred to as a stimulus sequence.

Another potential difference between this experiment and experiments investigating implicit learning was the emphasis on speed. Perhaps subjects focus on responses when they believe that they must perform quickly (which the typical implicit instructions emphasize), but focus on stimuli when they believe that accuracy is paramount. They may conclude that accuracy is especially important when told to learn the sequence explicitly. To investigate this possibility, we used two sets of instructions that varied between subjects. One set emphasized speed: "It is very important that you make your responses as quickly as you can while learning the sequence well," whereas the other emphasized accuracy: "It is very important that you make accurate responses while learning the sequence well."

\section{Results and Discussion}

RTs during training were summarized by taking the median RT for each set of 12 trials, yielding eight medians per block. The mean of these eight medians was calculated, yielding a single mean RT for each subject for each trial block. Accuracy was measured as the proportion of correct responses for each block.

Two subjects in the accuracy condition were excluded from the analysis. One produced RTs that were over $3 S D$ s from the remaining subjects $(M>1,000 \mathrm{msec})$, and the other produced mean accuracy scores that were more than $3 S D$ s from the remaining subjects (mean accuracy, 86\%). Mean RTs for the remaining subjects are depicted in Figure 1 . There were 28 subjects in the accuracy instructions condition, and 29 subjects in the speed instructions condition.

Subjects in the speed condition did not respond reliably faster than subjects in the accuracy condition $(F<1.0)$, nor was there a reliable interaction $(F<1.0)$, but both groups responded more quickly with practice $[F(3,165)=$ $\left.71.3, M S_{\mathrm{e}}=1,823, p<.001\right]$. The accuracy datadepicted in Figure 2-showed marginal effects between conditions, with subjects in the accuracy condition marginally more accurate than those in the speed condition $\left[F(1,55)=3.29, M S_{\mathrm{e}}=.001, p=.075\right]$. The data also showed a marginal interaction between accuracy and trial block $\left[F(3,165)=2.42, M S_{\mathrm{e}}=.001, p=.068\right]$. Thus, if the speed and accuracy instructions had any impact on behavior, it was not robust.

The results of the interview replicated the results of Experiment 1 . Subjects were again coded into one of three categories: screen, response, or switch. Three subjects reported that they had started by coding stimuli, but then switched to coding fingers. To be conservative, we categorized these subjects as coding responses, although two of these subjects reported that they had learned the entire sequence in terms of stimuli before switching to learning responses. The overwhelming proportion (41 out of

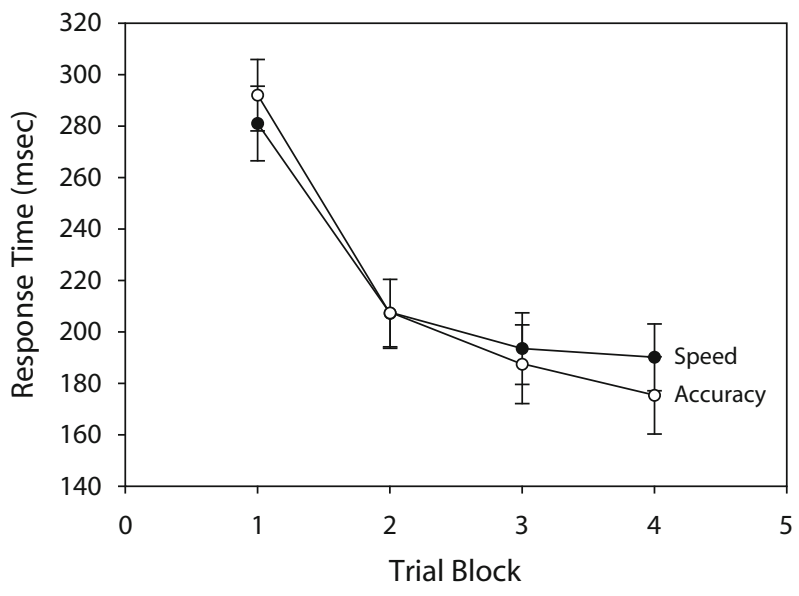

Figure 1. Response times during training across blocks for the speed and accuracy conditions, Experiment 3.

57) reported using the screen ( $p<.001$, two tailed by a binomial test). Instructions made no difference since the proportion of subjects reporting a screen coding was very close in both the speed instructions group (21 out of 28) and the accuracy group (20 out of 29). All of the subjects who reported coding responses claimed to have used finger movements, not button locations.

Nine subjects reported generating their own codes. Eight assigned tones or pitches to each key, and one claimed that rhythm was helpful. Of these 9 subjects who spontaneously selected their own code, 6 reported that the code corresponded to finger movements, and 3 said that the code corresponded to stimuli on the screen.

Although the instructions did not influence RTs, both groups responded quite quickly. Even in the initial trial block, their RTs averaged faster than $300 \mathrm{msec}$, which is faster than subjects typically respond at the end of training in the implicit SRTT. Thus, although the manipulation of instructions was not successful, we conclude that the potential difference in task emphasis - accuracy in this experiment, speed in implicit versions - does not account for the difference in what is learned.

Experiment 3 resolved some potential confounds from Experiment 1, but still used a verbal report measure. In Experiment 4, we sought evidence that explicit sequence knowledge is gained through stimuli when the dependent measure is response speed, as it is in the implicit version of the SRTT.

\section{EXPERIMENT 4}

Experiment 4 used the same methodology as that used in Experiment 2 to dissociate stimulus and response learning. Subjects were again asked to explicitly learn a sequence using an incompatible stimulus-response mapping. During training, the instructions emphasized speed, and subjects were told to learn the sequence explicitly to help them respond more quickly. At transfer, speed was again emphasized. The dependent measure of interest was whether subjects would respond more quickly when the stimuli matched 


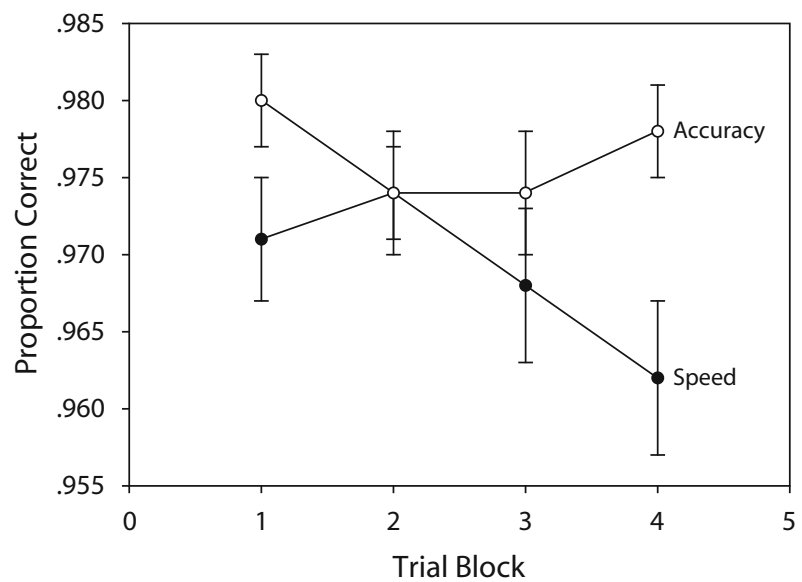

Figure 2. Accuracy during training across blocks for the speed and accuracy conditions, Experiment 3.

the training sequence (but the responses differed), or if the responses matched the training sequence (but the stimuli differed). We also included novel sequences at transfer as a baseline. We expected that subjects would respond the fastest to stimuli that matched the training sequence. Based on our prior work (Willingham \& Goedert-Eschmann, 1999), we also expected that subjects would show some RT advantage when the response sequence at transfer matched the training sequence; this advantage would be supported by procedural learning, not declarative.

\section{Method}

Subjects. Thirty-six undergraduate students $(5 \mathrm{male})$ from the University of Virginia participated in return for research credit in an introductory psychology course or for payment. All subjects reported normal hearing and normal or corrected-to-normal vision. Average subject age was 19.1 years (range, 18-24 years).

Stimuli and Apparatus. The stimuli and apparatus was the same as that used in Experiment 2. In order to ensure robust declarative knowledge, we used a six-element sequence with an RSI of $500 \mathrm{msec}$. The sequences were selected so that upon transfer when the mapping changed, the "stimulus" version would not inadvertently contain a segment of the "response" version of the sequence. Four sets of sequences were used. One sample set was: stimulus sequence $=124342$, response sequence $=231413$, novel sequence $=$ 312134.

Procedure. Instructions to subjects emphasized speed. They were told that the goal of the task was to respond as quickly as possible while making few errors. Subjects performed the task for 20 practice trials to familiarize them with the mapping and to allow them to ask questions about the procedure. After this practice, subjects were told that there was a six-element sequence embedded in the task and that they should try to identify it because it would help them respond more quickly. Subjects were alone during training. Training consisted of three trial blocks separated by a brief rest period, with four repetitions of the sequence in each block.

The experimenter returned after training and told the subject that he or she would now perform a new version of the task in which the stimulus-response mapping was compatible. Subjects practiced the new mapping for 20 trials with the experimenter present. Then subjects were told that they would perform three blocks of trials of this new task and that the sequence from the prior task would be embedded in at least one - and possibly more - of those blocks. Subjects were told that the goal of the task was to respond as quickly as possible and that identifying the sequence might help them to respond quickly. For all subjects, one transfer block used the same stimulus sequence as was used during training (but different responses), one block used the same response sequence as was used during training (but different stimuli), and one block was completely novel. The order of the blocks was counterbalanced across subjects.

\section{Results and Discussion}

The particular sequence set that was used had no effect in any analysis and is not further discussed. RTs during training were summarized by computing the median RT to each set of six contiguous trials. Mean RTs for subjects across blocks during training are shown in Figure 3. A repeated measures ANOVA showed a reliable decrease in RT $\left[F(11,242)=5.72, M S_{\mathrm{e}}=31,771, p<.001\right]$. Accuracy was summarized by taking the proportion correct for each trial block. Mean accuracy during training increased from $89.8 \%$ to $91.8 \%$ across the three trial blocks $[F(2,72)=$ $\left.4.87, M S_{\mathrm{e}}=0.003, p<.05\right]$. Note that accuracy is somewhat low because of the incompatible stimulus-response mapping.

The critical data are those from transfer, shown in Figure 4 . The order of the three sequence types had no effect in any analysis and is not further discussed. As is apparent from the figure, subjects responded faster with more training cycles $\left[F(3,105)=41.78, M S_{\mathrm{e}}=3,398, p<.001\right]$. There was also an overall difference in RT to the three sequence types (stimulus, response, novel) $[F(2,70)=6.05$, $\left.M S_{\mathrm{e}}=3,398, p<.01\right]$, but these main effects must be interpreted in light of an interaction of training cycles and sequence $\left[F(6,210)=3.10, M S_{\mathrm{e}}=2,107, p<.01\right]$.

Follow-up analyses showed that response sequence RTs were faster than novel RTs $\left[F(1,35)=8.07, M S_{\mathrm{e}}=\right.$ $5,207, p<.01]$, but that the improvement across cycles was equivalent $(F<1.0)$. On the basis of prior results, this advantage is likely supported by procedural learning, not declarative (Willingham, 1999; Willingham \& GoedertEschmann, 1999). Follow-up analyses for the stimulus sequence and response sequence RTs showed a reliable interaction of sequence type and block $[F(3,105)=5.30$, $\left.M S_{\mathrm{e}}=1,896, p<.01\right]$. As is clear from Figure 4, this in-

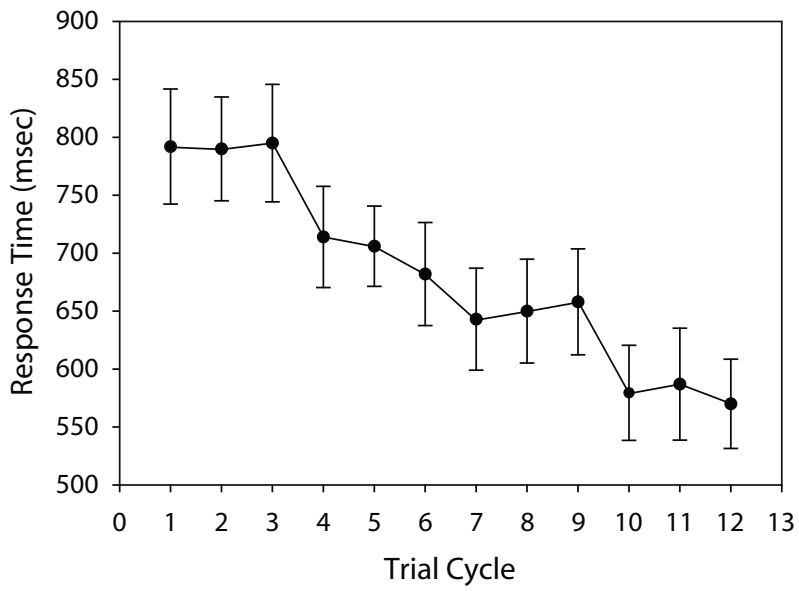

Figure 3. Response times during training across blocks, Experiment 4. 


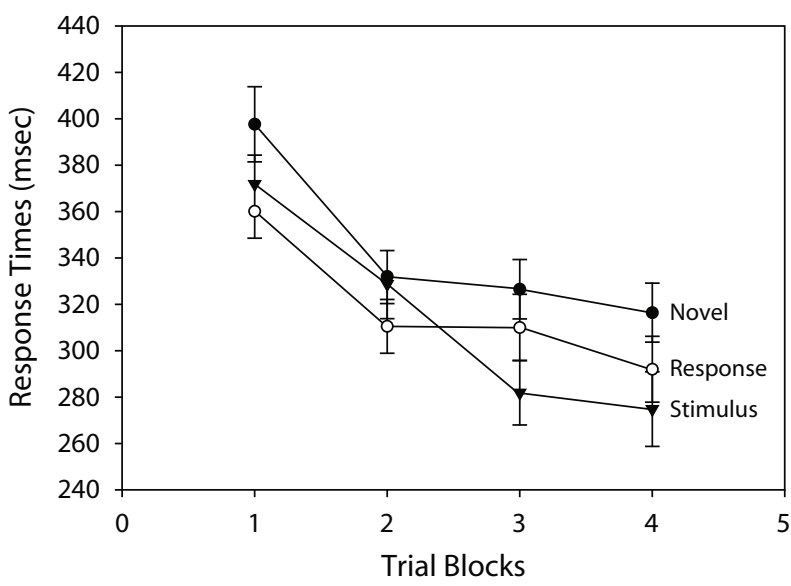

Figure 4. Response times during transfer in Experiment 4, where either the stimuli or responses matched the training sequence or the trials were novel.

teraction is due to stimulus RTs that are initially equivalent to response RTs $(p>.20)$, but that become faster in cycles 3 and $4\left[F(1,35)=4.46, M S_{\mathrm{e}}=6,472, p<.05\right]$.

The response sequence RTs are faster than the novel sequence RTs throughout transfer and are supported by procedural knowledge. Similar effects have been demonstrated in prior work (Willingham, 1999; Willingham \& Goedert-Eschmann, 1999). It is noteworthy that this advantage is observed in the very first cycle of response $\left[F(1,35)=5.28, M S_{\mathrm{e}}=4,357, p<.05\right]$. Subjects don't need to search for and notice the response sequencethe knowledge is recruited automatically and outside of awareness by the procedural system.

In contrast, the stimulus sequence shows no advantage over either condition on the first two cycles $(p s>.10)$, but it shows a significant RT advantage over the other two conditions in the third and fourth cycles. We interpret this pattern as reflecting a search process, whereby subjects seeking to use declarative knowledge must first detect the presence of the sequence before they can exploit it. Once they recruit explicit knowledge, the RT advantage is even greater than that afforded by implicit knowledge (see Curran \& Keele, 1993).

The performance advantage for the stimulus sequence is also observed in the accuracy data. Mean accuracy at transfer differed among the three conditions $[F(2,72)=$ $\left.3.16, M S_{\mathrm{e}}=.001, p<.05\right]$. A follow-up contrast showed that responses to the stimulus sequence $(M=.975, S D=$ $.040)$ were more accurate than responses to the response sequence $(M=.968, S D=.037)\left[F(1,72)=6.15, M S_{\mathrm{e}}=\right.$ $.001, p<.05]$. Accuracy for the novel group $(M=.968$, $S D=.032$ ) was not reliably different from either of the other two groups. Thus, subjects responded faster and more accurately to the stimulus sequence than to the response sequence.

\section{GENERAL DISCUSSION}

In four experiments, we have shown that subjects performing an explicit sequence learning task consistently code the sequence in terms of stimulus positions rather than response locations or effector movements. This generalization holds true for different dependent measures including self-report (Experiments 1 and 3), recognition memory (Experiment 2), and RT (Experiment 4).

One might suggest that the incompatible mapping used in Experiments 2 and 4 could cause response confusion for subjects and therefore encourage them to code stimuli. However, one could also make the argument that the incompatible mapping encourages subjects to focus on response locations, which would make them more likely to code the sequence in those terms; indeed, Clegg (2005) has made exactly that argument to account for previous findings showing that implicit sequence learning is coded in terms of response locations. We believe that the consistency of Experiments 1 and 3 (which used a compatible mapping) makes it unlikely that our results are an artifact of using an incompatible mapping.

We acknowledge, however, that these data do not allow claims of broad generalizability. It is possible that changes to the paradigm (e.g., instructions, length of training regimen) could yield changes in the coding scheme. It is also possible that the declarative system has flexibility in terms of coding (see, e.g., N. J. Cohen, Poldrack, \& Eichenbaum, 1997 ) and can change, depending on task requirements or the subject's goals.

Acknowledging these limitations, what can be said of memory systems? Little is known about memory system interaction in humans. A good bit more is known about memory system interaction in the rat. Three principles may be described (White \& McDonald, 2002): (1) There are multiple memory systems in the rat, (2) these systems learn simultaneously, and (3) each system is biased toward learning a different variety of information in the environment.

Following Poldrack and Packard (2003), we suggest that these three principles are applicable to human motor skill learning. Most researchers of human memory agree with the first principle, that human memory is supported by multiple systems (for a variety of perspectives, see Foster \& Jelicic, 1999; Schacter \& Tulving, 1994). In other work (Willingham \& Goedert-Eschmann, 1999; Willingham, Salidis, \& Gabrieli, 2002) we have presented data pertinent to the second principle, arguing that declarative and procedural learning of motor sequences occur in parallel. The work reported here-in conjunction with prior work (Willingham, 1999; Willingham, Wells, Farrell, \& Stemwedel, 2000) - suggests that declarative and procedural systems are attuned to different sorts of information, as suggested by the third principle. The memory system that learns motor sequences procedurally appears biased toward learning locations for motor response. The present experiments indicate that in contrast, the declarative system is biased toward learning motor sequences as a sequence of stimulus positions. This distinction is not synonymous with allocentric space and egocentric space, an orthogonal distinction. It is quite possible to learn either stimulus or response positions in either spatial frame of reference.

The present work complements work in other cognitive skill domains. Work by Ashby and his colleagues (Ashby, 
Maddox, \& Bohil, 2002) has indicated that declarative and procedural learning entail fundamentally different forms of representation in a categorization task, and has also supported different neuropsychological bases for these distinct processes (Ashby \& Spiering, 2004).

Another feature of memory concerns how these different systems affect one another. That feature remains unknown. It is possible that direct anatomic connections afford cooperation or competition among systems, or that each system may learn the information in the environment for which it is attuned, and the behavior of the animal is a product of some contribution of each system along a final common pathway (for reviews, see Gold, McIntyre, McNay, Stefani, \& Korol, 2001; Kesner, 1998).

This aspect of memory is also poorly understood in humans. We have presented some evidence that declarative and procedural learning do not directly affect one another; procedural learning is equivalent whether it is learned alone or in concert with declarative knowledge (Willingham \& Goedert-Eschmann, 1999). We have also suggested that they may affect one another indirectly through behavior.

In contrast, Poldrack and his colleagues (Poldrack et al., 2001; Poldrack, Prabhakaran, Seger, \& Gabrieli, 1999; Poldrack \& Rodriguez, 2004) have pointed to brain imaging data to argue that declarative learning and procedural learning are mutually inhibitory in a probabilistic categorization task. Declarative learning was associated with activation of medial temporal structures and deactivation of caudate; the opposite pattern was observed during procedural learning. The researchers argued that the two types of learning are incompatible; thus, if one sort of learning is occurring, the other is inhibited.

It is of course possible that the mode of interaction is different for probabilistic categorization and for motor sequence learning. Clarification of these differences awaits future research.

\section{AUTHOR NOTE}

R.K. is now at the Department of Psychology, Virginia Polytechnic Institute, Blacksburg, Virginia. This work was supported by Grants R01 NS040106-05, R01 MH065598-01A1, and R01 AG24106-01A1. Correspondence concerning this article should be addressed to D. T. Willingham, Department of Psychology, P.O. Box 400400, 102 Gilmer Hall, University of Virginia, Charlottesville, VA 22904-4400 (e-mail: willingham@virginia.edu).

Note-This article was accepted by the previous editorial team, when Colin M. MacLeod was Editor.

\section{REFERENCES}

Ashby, F. G., Maddox, W. T., \& BohIL, C. J. (2002). Observational versus feedback training in rule-based and information-integration category learning. Memory \& Cognition, 30, 666-677.

Ashby, F. G., \& Spiering, B. J. (2004). The neurobiology of category learning. Behavioral \& Cognitive Neuroscience Reviews, $\mathbf{3}$, 101-113.

ClEGG, B. A. (2005). Stimulus-specific sequence representation in serial reaction time tasks. Quarterly Journal of Experimental Psychology, 58A, 1087-1101.
Cohen, A., Ivry, R. I., \& Keele, S. W. (1990). Attention and structure in sequence learning. Journal of Experimental Psychology: Learning, Memory, \& Cognition, 16, 17-30.

Cohen, N. J., Poldrack, R. A., \& Eichenbaum, H. (1997). Memory for items and memory for relations in the procedural/declarative memory framework. Memory, 5, 131-178.

Cohen, N. J., \& SQuire, L. R. (1980). Preserved learning and retention of pattern-analyzing skill in amnesia: Dissociation of knowing how and knowing that. Science, 210, 207-210.

Curran, T., \& Keele, S. W. (1993). Attentional and nonattentional forms of sequence learning. Journal of Experimental Psychology: Learning, Memory, \& Cognition, 19, 189-202.

Foster, J. K., \& Jelicic, M. (Eds.) (1999). Memory: Systems, process, or function? Oxford: Oxford University Press.

GabRIELI, J. D. E. (1998). Cognitive neuroscience of human memory. Annual Review of Psychology, 4, 87-115.

Gold, P. E., McIntyre, C., McNay, E., Stefani, M., \& Korol, D. L. (2001). Neurochemical referees of dueling memory systems. In P. E Gold \& W. T. Greenough (Eds.), Memory consolidation: Essays in honor of James L. McGaugh (pp. 219-248). Washington, DC: American Psychological Association.

GoschKe, T. (1998). Implicit learning of perceptual and motor sequences: Evidence for independent learning systems. In P. A. Frensch (Ed.), Handbook of implicit learning (pp. 401-444). New York: Sage.

GRAF, P., \& SCHACTER, D. L. (1985). Implicit and explicit memory for new associations in normal and amnesic subjects. Journal of Experimental Psychology: Learning, Memory, \& Cognition, 11, 501-518.

Grafton, S. T., Hazeltine, E., \& Ivry, R. B. (1998). Abstract and effector-specific representations of motor sequences identified with PET. Journal of Neuroscience, 18, 9420-9428.

Grafton, S. T., SAlidis, J., \& Willingham, D. B. (2001). Motor learning of compatible and incompatible visuomotor maps. Journal of Cognitive Neuroscience, 13, 217-231.

Howard, J. H., MutTer, S. A., \& Howard, D. V. (1992). Serial pattern learning by event observation. Journal of Experimental Psychology: Learning, Memory, \& Cognition, 18, 1029-1039.

Jiménez, L., \& Méndez, C. (1999). Which attention is needed for implicit sequence learning? Journal of Experimental Psychology: Learning, Memory, \& Cognition, 25, 236-259.

Keele, S. W., Jennings, P., Jones, S., Caulton, D., \& Cohen, A. (1995). On the modularity of sequence representation. Journal of Motor Behavior, 27, 17-30.

KeSNER, R. P. (1998). Neurobiological views of memory. In R. P. Kesner (Ed.), Neurobiology of learning and memory (pp. 361-416). San Diego: Academic Press.

Koch, I., \& Hoffmann, J. (2000). The role of stimulus-based and response-based spatial information in sequence learning. Journal of Experimental Psychology: Learning, Memory, \& Cognition, 26, 863-882.

MAYR, U. (1996). Spatial attention and implicit sequence learning: Evidence for independent learning of spatial and nonspatial sequences. Journal of Experimental Psychology: Learning, Memory, \& Cognition, 22, 350-364.

NATTKEMPER, D., \& PRINZ, W. (1997). Stimulus and response anticipation in a serial reaction time task. Psychological Research, 60, 98-112.

Nissen, M. J., \& Bullemer, P. (1987). Attentional requirements of learning: Evidence from performance measures. Cognitive Psychology, 19, 1-32.

Poldrack, R. A., Clark, J., Paré-Blagoev, E. J., Shohamy, D., Moyano, J. C., Myers, C., \& GlucK, M. A. (2001). Interactive memory systems in the human brain. Nature, 414, 546-550.

PoldRACK, R. A., \& PACKARD, M. G. (2003). Competition among multiple memory systems: Converging evidence from animal and human brain studies. Neuropsychologia, 41, 245-251.

Poldrack, R. A., Prabhakaran, V., Seger, C. A., \& Gabrieli, J. D. E. (1999). Striatal activation during acquisition of a cognitive skill. Neuropsychology, 13, 564-574.

Poldrack, R. A., \& Rodriguez, P. (2004). How do memory systems interact? Evidence from human classification learning. Neurobiology of Learning \& Memory, 82, 324-332.

Reber, P. J., \& SQuire, L. R. (1998). Encapsulation of implicit and explicit memory in sequence learning. Journal of Cognitive Neuroscience, 10, 248-263. 
ReEd, J., \& Johnson, P. (1994). Assessing implicit learning with indirect tests: Determining what is learned about sequence structure Journal of Experimental Psychology: Learning, Memory, \& Cognition, 20, 585-594.

Remillard, G. (2003). Pure perceptual-based sequence learning. Journal of Experimental Psychology: Learning, Memory, \& Cognition, 29, 581-597.

Schacter, D. L., \& Tulving, E. (EDs.) (1994). Memory systems 1994. Cambridge, MA: MIT Press.

SQUiRE, L. R. (1992). Memory and the hippocampus: A synthesis from findings with rats, monkeys, and humans. Psychological Review, 99 , 195-231.

White, N. M., \& McDonald, R. J. (2002). Multiple parallel memory systems in the brain of the rat. Neurobiology of Learning \& Memory, 77, 125-184.

WiLlingham, D. B. (1998). A neuropsychological theory of motor skill learning. Psychological Review, 105, 558-584.

Willingham, D. B. (1999). Implicit motor sequence learning is not purely perceptual. Memory \& Cognition, 27, 561-572.

Willingham, D. B., \& Goedert-Eschmann, K. (1999). The relation between implicit and explicit learning: Evidence for parallel development. Psychological Science, 10, 531-534.
Willingham, D. B., Nissen, M. J., \& Bullemer, P. (1989). On the development of procedural knowledge. Journal of Experimental Psychology: Learning, Memory, \& Cognition, 15, 1047-1060.

Willingham, D. B., Salidis, J., \& Gabrieli, J. D. E. (2002). Direct comparison of neural systems mediating conscious and unconscious skill learning. Journal of Neurophysiology, 88, 1451-1460.

Willingham, D. B., Wells, L. A., Farrell, J. M., \& Stemwedel, M. E. (2000). Implicit motor sequence learning is represented in response locations. Memory \& Cognition, 28, 366-375.

ZIESSLER, M. (1994). The impact of motor responses on serial-pattern learning. Psychological Research, 57, 30-41.

ZIESSLER, M. (1998). Response-effect learning as a major component of implicit serial learning. Journal of Experimental Psychology: Learning, Memory, \& Cognition, 24, 962-978.

ZIESSLER, M., \& NATTKEMPER, D. (2001). Learning of event sequences is based on response-effect learning: Further evidence from a serial reaction task. Journal of Experimental Psychology: Learning, Memory, \& Cognition, 27, 595-613.

(Manuscript received July 24, 2003;

revision accepted for publication November 13, 2005.) 